\title{
Phase locking and periodic evolution of solitons in passively mode-locked fiber lasers with a semiconductor saturable absorber
}

\author{
N. N. Akhmediev \\ Australian Photonics Cooperative Research Centre, Optical Sciences Centre, The Australian National University, \\ Canberra, ACT 0200, Australia \\ J. M. Soto-Crespo \\ Instituto de Óptica, Consejo Superior de Investigaciones Cientificas, Serrano 121, 28006 Madrid, Spain
}

\section{S. T. Cundiff*}

Bell Laboratories, Lucent Technologies, Holmdel, New Jersey 07733

\section{B. C. Collings}

Bell Laboratories, Lucent Technologies, Holmdel, New Jersey 07733, and Department of Electrical Engineering, Princeton University, Princeton, New Jersey 08544

\section{W. H. Knox}

Bell Laboratories, Lucent Technologies, Holmdel, New Jersey 07733

Received January 20, 1998

\begin{abstract}
Passively mode-locked lasers with intracavity weakly birefringent fiber are theoretically analyzed based on two coupled complex one-dimensional Ginzburg-Landau equations. The model includes fiber birefringence, spectral filtering, saturable gain, and saturable loss. Phase-locked soliton solutions are found for small amounts of birefringence and several types of soliton with periodic polarization evolution for higher amounts of birefringence. Numerical simulations show qualitative agreement with experimental results. ( 1998 Optical Society of America

OCIS codes: $\quad 060.4370,060.5530,060.7140$
\end{abstract}

Passively mode-locked fiber lasers ${ }^{1-11}$ are unique sources of ultrashort optical pulses for telecommunications and other applications. Ultrashort-pulse generation in fiber lasers is based on a variety of schemes, including coupled cavities, ${ }^{1,5}$ fast saturable absorbers, ${ }^{3}$ additive-pulse mode locking, ${ }^{6}$ and nonlinear polarization rotation. $^{7-9}$ Semiconductor mirror-saturable absorbers have been used in a variety of mode-locked lasers. ${ }^{10,11}$ Birefringent elements in the cavity are of special interest because they can give rise to new phenomena. Phase locking of the two orthogonal polarization components of a soliton owing to nonlinear effects' overcoming the linear beating in a weakly birefringent, lossless fiber was theoretically predicted. ${ }^{12,13}$ Polarization-locking effects were experimentally discovered ${ }^{14}$ in a laser with birefringent fibers and a semiconductor saturable absorber but without an explicit polarizer. Although there are similarities between these, it is not immediately obvious that the theoretical results apply to a system that incorporates gain and (saturable) loss.

Our aim here is to relate the phenomena predicted in Refs. 12 and 13 to those discovered experimentally in
Ref. 14. These phenomena are similar in that in both cases the phase-locking effect is due to the nonlinearity in the birefringent fiber. However, in the theoretical research ${ }^{12,13}$ the phase locking happens in a Hamiltonian system in which, for a given set of experimental parameters, there exists a one-parameter family of soliton solutions. In the experimental observations dissipative effects play an important role in the pulse propagation inside the laser cavity, fixing the amplitude, width, and shape of the soliton. The pulse profile then depends on the parameters that describe the dissipative effects in the cavity. Here we investigate the behavior of pulses in this system as the linear birefringence in the cavity is varied. We have found, in particular, that there exist at least four different branches of soliton solutions. For one of these branches the two polarization components are locked in phase. For the other branches the polarization state evolves periodically.

A laser with birefringent fiber elements in the cavity and with a saturable absorber can be modeled as a distributed system that is governed by the following two coupled modified nonlinear Schrödinger equations with nonlinear, nonlocal in time, and nonconservative terms ${ }^{15}$ :

(C) 1998 Optical Society of America 


$$
\begin{gathered}
i \phi_{z}+\gamma \phi+\frac{D}{2} \phi_{t t}+|\phi|^{2} \phi+A|\psi|^{2} \phi+B \psi^{2} \phi^{*} \\
=i\left[g\left(Q_{1}\right)-\delta_{s}\left(\left|\phi^{2}\right|\right)\right] \phi+i \beta \phi_{t t} \\
i \psi_{z}-\gamma \psi+\frac{D}{2} \psi_{t t}+|\psi|^{2} \psi+A|\phi|^{2} \psi+B \phi^{2} \psi^{*} \\
=i\left[g\left(Q_{2}\right)-\delta_{s}\left(|\psi|^{2}\right)\right] \psi+i \beta \psi_{t t}
\end{gathered}
$$

where $z$ is normalized to the cavity length $Z_{0} ; t$ is the normalized retarded time $t=\left\{T-\left[z /\left(V_{g} Z_{0}\right)\right]\right\} / T_{0}$ $\left(T_{0}=\sqrt{Z_{0}\left|\beta_{2}\right|}\right) ; \beta_{2}$ is the intracavity group-velocity dispersion, $\psi$ and $\phi$ are the normalized envelopes of the two optical field components; $\gamma$ is half-difference between the propagation constants of the two components of the field; $D=\beta_{2} /\left|\beta_{2}\right| ; A$ is the cross-phase modulation coefficient; $B$ is the coefficient of the energyexchange term (four-wave mixing); $\beta$ represents spectral filtering $(\beta>0) ; g\left(Q_{i}\right)$ is the gain in the cavity, which depends on the energy, $Q_{1}=\int_{-\infty}^{\infty}\left(|\phi|^{2}\right) \mathrm{d} t$, $Q_{2}=\int_{-\infty}^{\infty}\left(|\psi|^{2}\right) \mathrm{d} t$; and $\delta_{s}$ is the total loss, including loss that is due to a semiconductor saturable absorber.

In the cavity used in the research reported in Ref. 14, two sections of the fiber that made up the laser cavity were wrapped around the paddles of a polarization controller. The bending of the fiber produced birefringence with well-defined axes in these sections. The relative angles of the birefringence axes to each other as well as to the rest of the cavity were varied by changes in the angles $\theta_{1}$ and $\theta_{2}$ of the paddles (see Fig. 1 of Ref. 14). Each of these pieces can be described by a $2 \times 2$ matrix. Multiplying the matrices of all cavity elements and diagonalizing the resulting matrix, we find the axes and the amount of birefringence for the whole cavity. Equations (1) are written on this basis; $\gamma$ gives the half-difference between the propagation constants of the two polarization components. Although there is relatively large birefringence in the sections of the cavity that are wrapped around the polarization controller, the net phase shift between the two components per round trip is of the order of one wavelength. This justifies the use of the above analysis, which applies to the case of low birefringence. For high birefringence, qualitatively different behavior can occur. ${ }^{16}$

The gain term $g\left(Q_{i}\right)$ in Eqs. (1) describes an active medium with a recovery time much longer than the round-trip time of the cavity and therefore does not depend explicitly on $t$. It describes depletion of the gain medium and depends on the partial pulse energy

$$
g\left(Q_{i}\right)=\frac{g_{0}}{1+Q_{i} / E_{L}},
$$

where $g_{0}$ is the small-signal gain and $E_{L}$ is the saturation energy. The absorption in the semiconductor is described by the following rate equation ${ }^{10}$ :

$$
\frac{\partial \delta_{s}}{\partial t}=-\frac{\delta_{s}-\delta_{0}}{T_{1}}-\frac{|\Upsilon|^{2}}{E_{A}} \delta_{s}
$$

where $T_{1}$ is the recovery time of the saturable absorber, $\delta_{0}$ is the loss introduced by the absorber in absence of pulses, $Y$ refers to $\psi$ or $\phi$, and $E_{A}$ is the saturation energy of the absorber.
The solution of the latter equation is ${ }^{17}$

$$
\delta_{s}(t)=\delta_{0} f^{-1}(t)\left[\frac{1}{T_{1}} \int_{-\infty}^{t} f\left(t^{\prime}\right) \mathrm{d} t^{\prime}+1\right],
$$

where

$$
f(t)=\exp \left[\int_{-\infty}^{t}\left(\frac{1}{T_{1}}+\frac{|Y|^{2}}{E_{A}}\right) \mathrm{d} t\right]
$$

Our numerical results show that for small values of the birefringence parameter $\gamma$ the two polarization components are phase locked, but they are not necessarily equal (see Fig. 1). The point on the Poincaré sphere that corresponds to this state is close to the north pole but does not coincide with it [or equivalently to the south pole, because if $(\phi, \psi)$ is a solution, then $(\phi,-\psi)$ is also a solution]. The energy $Q$ versus $\gamma$ for these states is represented by the upper dotted curve in Fig. 2. In addition to these phase-locked states, there are solutions for which the state of polarization evolves periodically, i.e., phase locking is absent (see the trajectories on the Poincaré spheres in Fig. 2). There are at least three qualitatively different types of solution with different trajectories on the Poincaré sphere. Their corresponding curves $Q$ versus $\gamma$ are located separately in the diagram in Fig. 2. Almost all solitons that are described by these curves are stable. The results shown in Figs. 1 and 2 were obtained for $E_{L}=2, \delta_{0}=0.15, T_{1}=3, \beta=0.02, g_{0}=0.2, E_{A}=1$, $A=2 / 3, B=1 / 3$, and $D=+1$. Similar results were obtained for other values of the gain and loss parameters, although the locations of the soliton branches on the $Q-\gamma$ diagram changed.

The calculated polarization-evolution frequency (PEF) for the phase-locked solutions and for type II periodic solitons is shown in Fig. 3(a). To calculate the frequency, we scaled our dimensionless magnitudes, taking $Z_{0}=4 \mathrm{~m}, \beta_{2}=16 \mathrm{ps}^{2} / \mathrm{km}$, so that time scaling is $T_{0}=253 \mathrm{fs}$ and the cavity round-trip time is $\approx 0.02 \mu \mathrm{s}$. Figure 3 shows that switching between the two soliton states happens at approximately $\gamma=0.14$.

The experimental PEF as a function of $\gamma$ is shown in Fig. 3(b). ${ }^{14}$ The PEF measured for $\mathrm{cw}$ operation is used to estimate $\gamma$. The PEF is directly related to the beat length, which in turn is related to $\gamma$. The transition from polarization locked to unlocked states is in

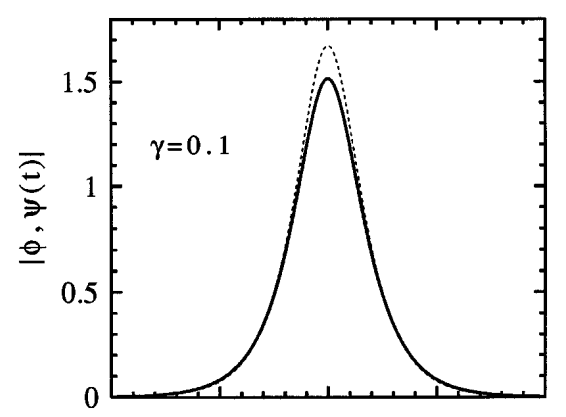

Fig. 1. Amplitude profiles for the two components of the phase-locked solutions. Solid curve, $\phi$; dashed curve, $\psi$. 

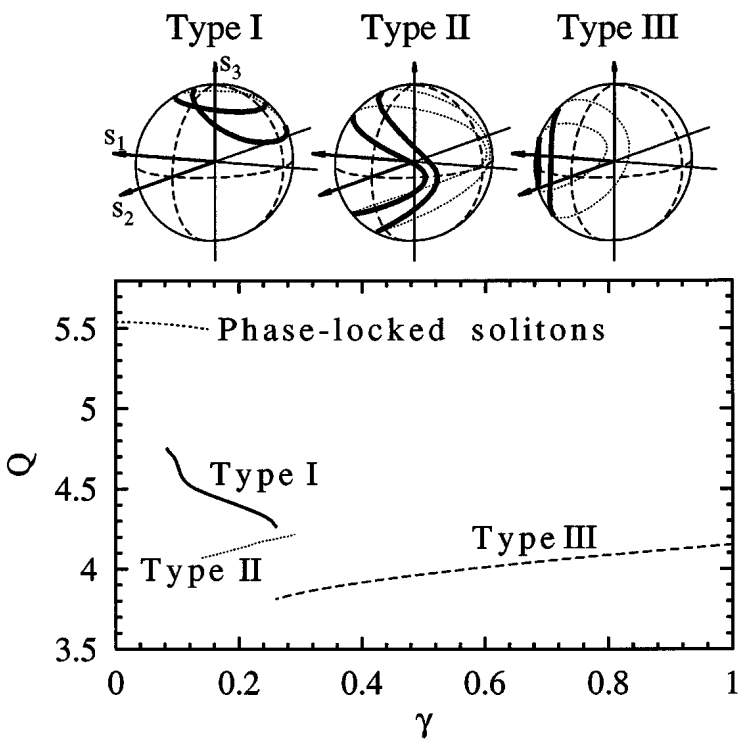

Fig. 2. Pulse energy $Q$ versus $\gamma$ for four qualitatively different types of soliton. The two polarization components are phase locked in the interval $0<\gamma<0.16$ (upper dotted curve). The state of polarization is elliptical and can be represented by a dot on the Poincaré sphere that is close to the north or south pole. The periodic evolution of the state of polarization on the Poincaré sphere for other solutions is shown above each curve. In the case of the other three kinds of solution with lower values of $Q$, the state of polarization evolves periodically. In each sphere we show two trajectories that correspond to the two solutions for the limiting values of $\gamma$ in their corresponding curves $Q(\gamma)$.
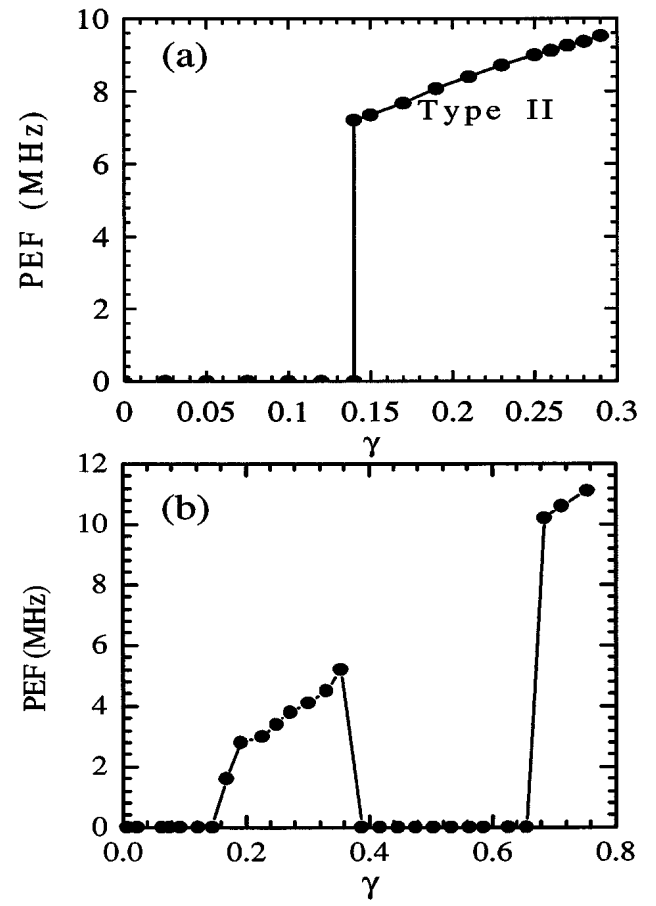

Fig. 3. (a) Calculated PEF for phase-locked solitons and for the type II periodic solitons in Fig. 2. (b) Experimentally observed PEF.

qualitative agreement between theory and experiment, in which switching occurs at approximately $\gamma=0.17$.
In our model the main control parameter is the linear birefringence, as in the experiments. ${ }^{14}$ The pulse energy is the parameter that self-adjusts to a given $\gamma$. Numerical simulations show that there are at least four different branches of solitons. There are phase-locked solutions at small values of the linear birefringence and relatively high values of the pulse energy. In addition, there are three branches of dynamic solitons with qualitatively different types of evolution of the state of polarization. These appear at higher values of $\gamma$ and lower values of $Q$.

In experimental results presented in Fig. 3(b), there is a second region of polarization locking for larger values of $\gamma$ that does not appear in the theoretical results. We ascribe this to the presence of several sections of higher-birefringence fiber within the experimental laser cavity. Preliminary theoretical investigation showed that phase locking is also present in such a cavity. Determining whether this phase locking results in the experimentally observed multiple locking regions will require a much more extensive investigation.

The research of J. M. Soto-Crespo was supported by Comunidad de Madrid contract 06T/039/96 and by Dirección Generale de Enseñanza Superior contract PB96-0819.

*Present address, JILA, University of Colorado and National Institute of Standards and Technology, Boulder, Colorado 80309-0440.

\section{References}

1. I. N. Duling, Opt. Lett. 16, 539 (1991).

2. C.-J. Chen, P. K. A. Wai, and C. R. Menyuk, Opt. Lett. 19, 198 (1994).

3. H. A. Haus, J. G. Fujimoto, and E. P. Ippen, J. Opt. Soc. Am. B 10, 2068 (1991).

4. D. U. Noske, N. Pandit, and J. R. Taylor, Electron. Lett. 28, 2185 (1992).

5. P. A. Belanger, J. Opt. Soc. Am. B 8, 2077 (1991).

6. H. A. Haus, E. P. Ippen, and K. Tamura, IEEE J. Quantum Electron. 30, 200 (1994).

7. V. J. Matsas, D. J. Richardson, T. P. Newson, and D. N. Payne, Opt. Lett. 18, 358 (1993).

8. M. Hofer, M. E. Fermann, F. Haberl, M. H. Ober, and A. J. Schmidt, Opt. Lett. 16, 502 (1991).

9. L. E. Nelson, D. J. Jones, K. Tamura, H. A. Haus, and E. P. Ippen, Appl. Phys. B 65, 277 (1997).

10. F. X. Kärtner, I. D. Jung, and U. Keller, IEEE J. Sel. Topics Quantum Electron. 2, 540 (1996).

11. B. C. Collings, K. Bergman, S. T. Cundiff, S. Tsuda, N. Kutz, J. E. Cunningham, W. Y. Jan, and W. H. Knox, IEEE J. Sel. Topics Quantum Electron. 3, 1065 (1997).

12. N. N. Akhmediev, A. V. Buryak, and J. M. Soto-Crespo, Opt. Commun. 112, 278 (1994).

13. N. N. Akhmediev, A. V. Buryak, J. M. Soto-Crespo, and D. R. Andersen, J. Opt. Soc. Am. B 12, 434 (1995).

14. S. T. Cundiff, B. C. Collings, and W. H. Knox, Opt. Express 1, 12 (1997); www.osa.org.

15. N. N. Akhmediev and A. Ankiewicz, Solitons, Nonlinear Pulses and Beams (Chapman \& Hall, New York, 1997).

16. P. K. A. Wai, C. R. Menyuk, and H. H. Chen, Opt. Lett. 16, 1231 (1991).

17. N. N. Akhmediev, A. Ankiewicz, M. J. Lederer, and B. Luther-Davies, Opt. Lett. 23, 280 (1998). 